\title{
A novel ultrasound scanning approach for evaluating femoral cartilage defects of the knee: comparison with routine magnetic resonance imaging
}

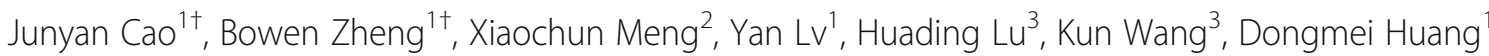
and Jie Ren ${ }^{1 *}$

\begin{abstract}
Background: This study aimed to assess a novel ultrasound (US) scanning approach in evaluating knee femoral cartilaginous defects, compared with magnetic resonance imaging (MRI, commonly used for knee imaging) and arthroscopy (gold standard).

Methods: Sixty-four consecutive patients (65 knees) were prospectively evaluated between April 2010 and July 2011.

Results: The overall sensitivity (62.2 and 69.4\%), specificity (92.9 and 90.5\%), accuracy (75.4 and 78.5\%), and adjusted positive (88.7 and 90.4\%) and negative predictive (69.5 and 73.3\%) were similar for both radiologists (weighted $k=0.76$ ). Furthermore, agreement between grading by US and MRI was substantial (weighted $k=0.61$ ).

Conclusions: In conclusion, the novel US scanning approach allows similar diagnostic performance compared to routine MRI for knee cartilage defects. US is more accessible, easier to perform, and less expensive than MRI, with potential advantages of easier initial screening and assessment of cartilage defects.
\end{abstract}

Keywords: Cartilage disease, Ultrasound, Magnetic resonance imaging, Diagnostic performance, Arthroscopy, Sensitivity, Specificity

\section{Background}

Progressive articular cartilage defects in the knee are a major cause of pain, disability, and medical expenses in the general population and particularly in the elderly [1]. Precise assessment of cartilaginous abnormality is important to determine the appropriate treatments, e.g., osteotomy, mosaicplasty, drugs, and autologous chondrocyte transplantation [2]. Ultrasound (US) is widely available and relatively inexpensive and has proven to be a useful modality for the routine clinical assessment of joint diseases [3]. US is radiation-free and non-invasive and allows dynamic assessment of moving structures [4].

\footnotetext{
* Correspondence: renjieguangzhou@126.com

Junyan Cao and Bowen Zheng share the first authorship.

†Junyan Cao and Bowen Zheng contributed equally to this work.

'Department of Medical Ultrasonics, The Third Affiliated Hospital of Sun

Yat-sen University, 600 Tianhe Road, Guangzhou 510630, People's Republic of

China

Full list of author information is available at the end of the article
}

Previous studies evaluated the feasibility and diagnostic value of US for detecting cartilaginous defects [5-15]. Previous studies reported associations of US with histologic and arthroscopic classifications of cartilage defects $[11,13]$, and with an acceptable diagnostic performance [14], suggesting that knee US is a promising technique for screening degenerative changes of articular cartilage in patients with osteoarthritis (OA) [14]. Nevertheless, the correlations observed in previous studies were low, with coefficients of $0.262-0.655[13,14]$ and could be due to the selection of the indicators, the selection of US systems, and the experience of the raters. In addition, negative predictive values (NPV) remained low (23.8$45.8 \%$ ), implying that a negative finding using US does not rule out cartilage degenerative changes [14]. Although a positive finding in US is a strong indicator of arthroscopic degenerative changes of cartilage [14], the

(c) The Author(s). 2018 Open Access This article is distributed under the terms of the Creative Commons Attribution 4.0 International License (http://creativecommons.org/licenses/by/4.0/), which permits unrestricted use, distribution, and 
technique still needs improvements before routine clinical use.

Previous assessments were performed with a transducer capable of the highest frequency available for routine clinical use, already resulting in the highest sensitivity possible [13, 14]. The diagnostic accuracy of US is often dependent upon the scanning approach in different US indications [16-18]. Therefore, we hypothesized that improvement of the scanning technique could enhance US diagnostic value. Indeed, previous studies used a fixed flexed knee (e.g., $120^{\circ}$ ) with transverse scanning only $[13,14]$, but the knee flexion angle influences the correlation between US and histologic classification of cartilage defects, although the optimal angle remains unknown [13]. In addition, since only transverse scanning was applied, it remains unclear whether longitudinal scanning could offer more benefits, especially when depicting the condyle [14].

Therefore, this study aimed to assess a novel US scanning approach in evaluating knee femoral cartilaginous defects, compared with magnetic resonance imaging (MRI, commonly used for knee imaging) and arthroscopy (gold standard).

\section{Methods}

\section{Study design and patients}

In this prospective study, 64 consecutive patients (65 knees) scheduled for knee arthroscopy between April 2010 and July 2011 were prospectively evaluated by US and MRI at our hospital. The study was approved by the research ethics committee of the Third Affiliated Hospital of Sun Yat-sen University. All patients signed an informed consent prior to participation in the study.

Patients with a chief complaint of knee pain or disability and scheduled for arthroscopy were enrolled in this study. The patients underwent arthroscopy of the knee because of suspicion of internal derangement. Patients with prior knee surgery (e.g., total knee arthroplasty) and contraindications to MRI were excluded.

\section{US examination}

US examination of the knee joint was conducted a week before arthroscopy using a LOGIQ 700 (GE Medical Systems, Milwaukee, WI, USA) with a $7-9-\mathrm{MHz}$ highresolution linear transducer. A novel US scanning approach based on the functional anatomy of the knee (Fig. 1) [15] was proposed, considering articular motions and both transverse and longitudinal scanning (Fig. 2). (1) In the supine position with a fully extended knee $\left(0^{\circ}\right)$, transverse and longitudinal scanning of bilateral sides of the patella for the anterior portions of both condyles was carried out (Fig. 2a). (2) In the prone position, longitudinal and transverse scanning of the popliteal fossa for the posterior portions of both condyles was

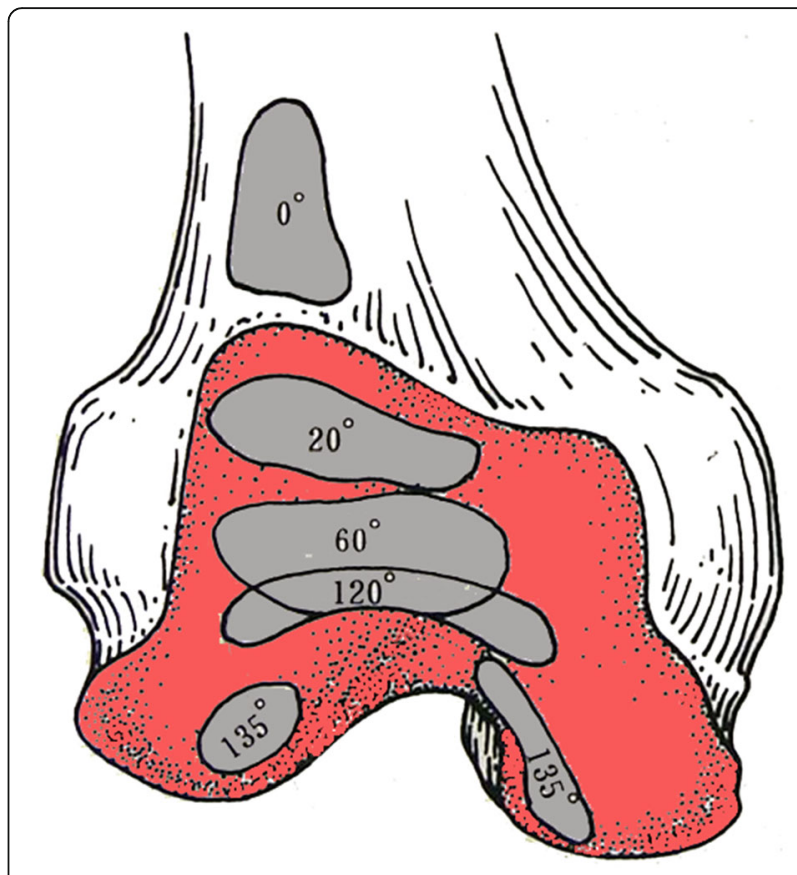

Fig. 1 Contact areas of the patellofemoral articulation during different arcs of motion, adapted from Shahriaree's O'Connor's Textbook of Arthroscopic Surgery [15]. Red area, femoral cartilage; gray areas, contact areas of the femoral cartilage of the patellofemoral articulation during different arcs of motion

performed (Fig. 2b). (3) In the supine position with maximum knee flexion, transverse and longitudinal scanning of the suprapatellar recess for the trochlear surface was undertaken (Fig. 2c). (4) Finally, in the same position, transverse and longitudinal scanning of bilateral sides of the patella was performed again, for the weight-bearing portion of the condyles (Fig. 2c). The US beam was always kept perpendicular to the femur surface. Maximum flexion angle of each patient was recorded, and an angle $>135^{\circ}$ was considered good flexion. This angle was confirmed using a lateral photography and the Angler app (an iOS app for angle measurement which was available only on Apple Store China but is unfortunately no longer available). The patient was in the supine position with maximum knee flexion, then a lateral photography was taken and the flexion angle was measured by this app.

A US grading system for knee femoral cartilage defects, based on the International Cartilage Repair Society (ICRS) classification [5, 7, 19, 20], was used: grade 0, normal cartilage; grade 1 , nearly normal cartilage with blurred margin or partial lack of clarity without thickness change; grade 2, abnormal cartilage with blurring or obliteration of the margin, lack of clarity, and overt local thinning of the cartilage ( $<50 \%$ of cartilage depth); grade 3 , severely abnormal cartilage with blurring and obliteration of the margin and obvious focal thinning for 


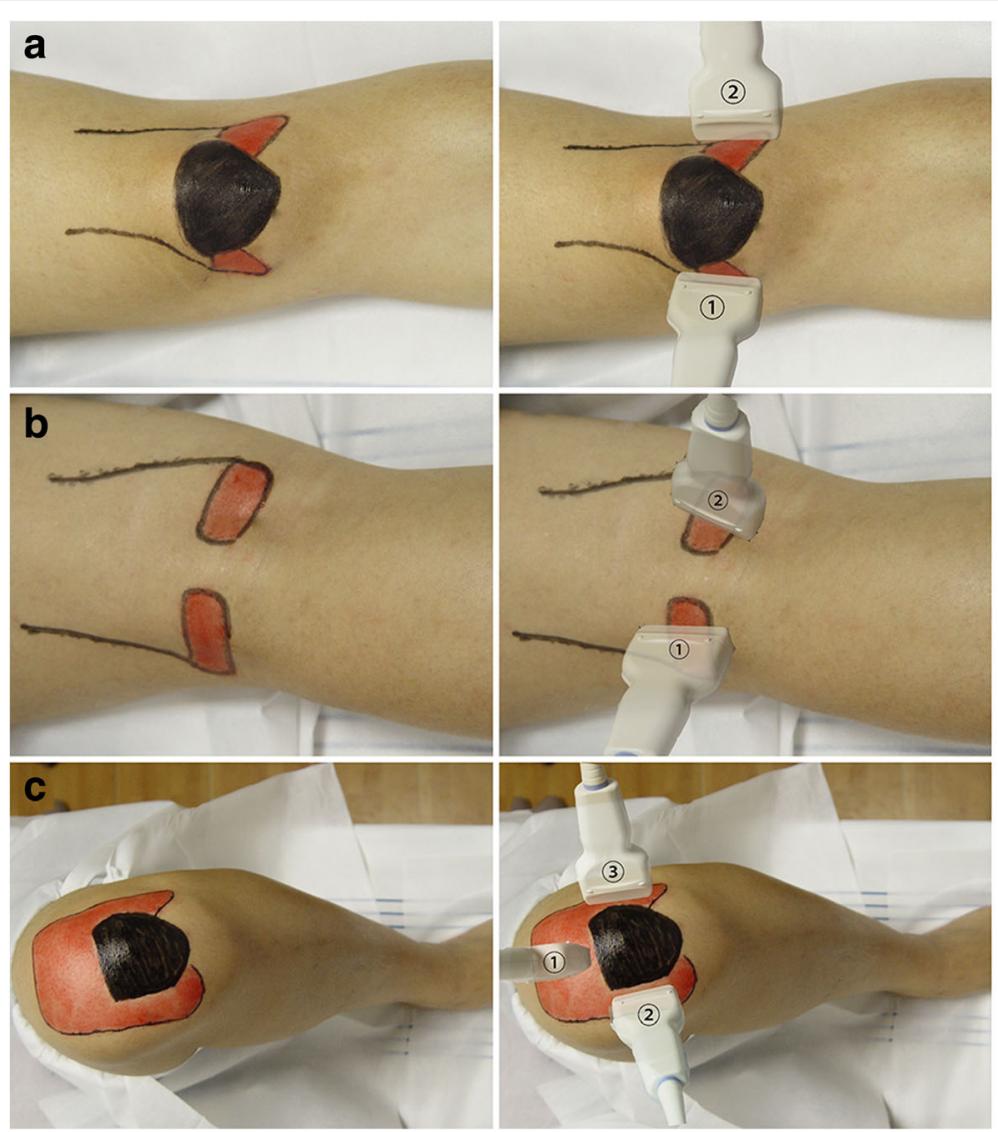

Fig. 2 Novel ultrasound (US) scanning approach for the detection of femoral cartilage. a With fully extended knee $\left(0^{\circ}\right)$, transverse and longitudinal scanning of bilateral sides of the patella for cartilage of the anterior portion of medial (1) and lateral (2) condyles with the patient in the supine position. $\mathbf{b}$ Transverse and longitudinal scanning of the popliteal fossa for the posterior portion of medial (1) and lateral (2) condyles with the patient in a prone position with a fully extended knee. c With maximum knee flexion in the supine position, transverse and longitudinal scanning of the trochlear surface (1) and weight-bearing portion of medial (2) and lateral (3) condyles. Red area, surface projection of the femoral cartilage; black area, surface projection of the patella

$>50 \%$ of the cartilage depth but intact cartilage-bone interface; and grade 4, severely abnormal cartilage with complete loss of cartilage and coarse or irregular cartilage-bone interface.

Two musculoskeletal radiologists (JYC and JR, with 5 and 15 years of clinical experience, respectively) were trained for 2 months to perform the novel examination method before the present study. This training involved ten volunteers. The radiologists repeatedly scanned those volunteers, alone and together, and compared their results. In this study, the radiologists scanned each patient immediately one after another (according to their availability, without a predefined order) and made assessments independently during real-time scanning, based on individual findings. A form with a schematic drawing of the articular surfaces of the femoral cartilage [19] was used. The trochlear surface and lateral and medial femoral condyles were evaluated separately. In the presence of multiple cartilaginous defects on the same articular surface, the worst score was attributed.

\section{MRI evaluation}

MRI examinations were performed within 1 week before arthroscopy on a Signa Excite 1.5 Tesla MR System (GE Medical Systems, Milwaukee, WI, USA), using a phased array knee coil. Patients were placed in the supine position with the knee fully extended. All MRI examinations consisted of sagittal T1- and T2-weighted, sagittal fatsaturated (fs) proton density (PD)-weighted, coronal fs PD-weighted, and axial fs PD-weighted fast spin echo (FSE) sequences. The imaging parameters are presented in Additional file 1: Table S1.

All MRI images were interpreted by one radiologist (XCM) with over 15 years of experience in musculoskeletal imaging. The radiologist was blinded to the clinical history and US findings. The cartilage was morphologically graded according to a modified ICRS classification 
system [19]: grade 0 , normal cartilage; grade 1 , cartilage with intact surface and no tissue loss, but fibrillation and superficial fissures; grade 2, deep defects, but $<50 \%$; grade 3 , lesions representing $>50 \%$ of the cartilage thickness; and grade 4, defects extending into the subchondral bone. The same schematic drawing of articular surfaces of the femoral cartilage was used to mark defect locations and degrees. The trochlear surface and lateral and medial femoral condyles were evaluated separately. In the presence of multiple cartilaginous defects on the same articular surface, the worst score was attributed.

\section{Arthroscopy}

Two orthopedic surgeons (HDL and KW, with 15 and 25 years of clinical experience, respectively) were involved in this study. They were blinded to the US and MRI findings. Knee arthroscopy with fluid irrigation was performed using the standard anterolateral and anteromedial portals to assess the articular cartilage defects and potential comorbidities. The surgeons were free to flex and extend the patients' knees. For cartilage evaluation by arthroscopy, the same schematic drawing of the femoral cartilage surfaces of the knee was used. The surgeons performed their assessment in consensus. The trochlear surface and lateral and medial femoral condyles were evaluated separately. The cartilage lesions were graded analogously to the US and MRI using the ICRS classification [19]. In the presence of multiple cartilaginous defects on the same articular surface, the worst score was attributed. Arthroscopic grading served as the gold standard.

\section{Statistical analysis}

With arthroscopic findings as a reference, sensitivity, specificity, and accuracy of US and MRI for femoral cartilage defects were assessed. In addition, positive predictive value (PPV) and NPV were calculated and adjusted for the prevalence of femoral cartilage lesions documented in arthroscopy at our institution (51.9\%), based on previous recommendations [21]. The threshold of femoral cartilage abnormality was set between grade 0 as negative and grades $1-4$ as positive. Furthermore, detection rates of each grade of cartilage defects and proportion of cartilage lesions graded identically and within one grade in arthroscopy and imaging modalities were calculated. The McNemar chi-square test was used to compare the groups.

Weighted $\kappa$ statistics for the level of agreement among different methods and between radiologists (US) were used [22]. The Student $t$ test was used to compare the $\kappa$ values between grades assigned by arthroscopy and US or MRI. Error analysis was performed for the first blinded US evaluation by the same radiologists after the revelation of arthroscopic findings. All false-positive or false-negative results were analyzed, and the most likely reason for the diagnostic error was recorded.
All statistical analyses were performed with SPSS 17.0 (IBM, Armonk, NY, USA) and STATA 11 (StataCorp LP, College Station, TX, USA). Two-sided $P$ values $<0.05$ were considered significantly significant.

\section{Results}

\section{Patient baseline characteristics and arthroscopy data}

Sixty-four consecutive patients (65 knees) with various abnormalities of the knee were assessed, including 21 men and 43 women, with a mean age of 42 years (range, 18-75). Final diagnoses included OA (25 knees), meniscus injury (11 knees), traumatic arthritis (11 knees), chondromalacia patella (7 knees), anterior cruciate ligament tears (6 knees), dislocation of the patella ( 3 knees), and meniscus cyst ( 2 knees). Among the 65 knees, 90.8\% $(59 / 65)$ had a good flexion. Altogether, 195 knee femoral cartilage surfaces, including the trochlear surface (TS), lateral femoral condyles (LC), and medial femoral condyles (MC), were evaluated by US, MRI, and arthroscopy. Arthroscopy revealed that 84, 20, 30, 38, and 23 surfaces had grades $0,1,2,3$, and 4 defects, respectively (Table 1). The distribution of cartilage defects identified by arthroscopy is shown in Table 1 .

\section{Overall diagnosis performances of US and MR}

Table 2 shows the overall sensitivity, specificity, accuracy, and crude and adjusted PPV and NPV for US and MRI in detecting knee femoral cartilage defects, using arthroscopy as the gold standard. Figures 3 presents typical cases of each grade of cartilage defects. The sensitivity (62.2 and 69.4\%), specificity (92.9 and 90.5\%), and accuracy (75.4 and $78.5 \%$ ) were similar between two independent radiologists performing the US. Interobserver agreement was obtained between the two radiologists, as indicated by a weighted $\kappa$ value of 0.76 . Considering the prevalence of cartilage lesions of $51.9 \%$ at our institution, obtained based on the analysis of 832 knee arthroscopies from January 2002 to December 2011, adjusted PPV was 88.7-90.4\% (false PPV $=9.6-11.3 \%$ ), and adjusted NPV was $69.5-73.3 \%$ (false NPV $=26.7-30.5 \%$ ). Only a lower sensitivity of US was observed for radiologist 1 (JYC, 5 years of clinical experience) compared with MRI $(P=$ 0.042). Other parameters showed non-significant

Table 1 Location and grades of cartilaginous defects in 65 knees by arthroscopy

\begin{tabular}{llllll}
\hline Surface & Grade 0 & Grade 1 & Grade 2 & Grade 3 & Grade 4 \\
\hline TS & $27(13.9)$ & $7(3.6)$ & $11(5.6)$ & $13(6.7)$ & $7(3.6)$ \\
MC & $25(12.8)$ & $7(3.6)$ & $11(5.6)$ & $14(7.2)$ & $8(4.1)$ \\
LC & $32(16.4)$ & $6(3.1)$ & $8(4.1)$ & $11(5.6)$ & $8(4.1)$ \\
Total & $84(43.1)$ & $20(10.3)$ & $30(15.3)$ & $38(19.5)$ & $23(11.8)$ \\
\hline
\end{tabular}

Data are the numbers of surfaces. Values in parentheses are percentages TS trochlear surface, MC medial condyles, LC lateral condyles 


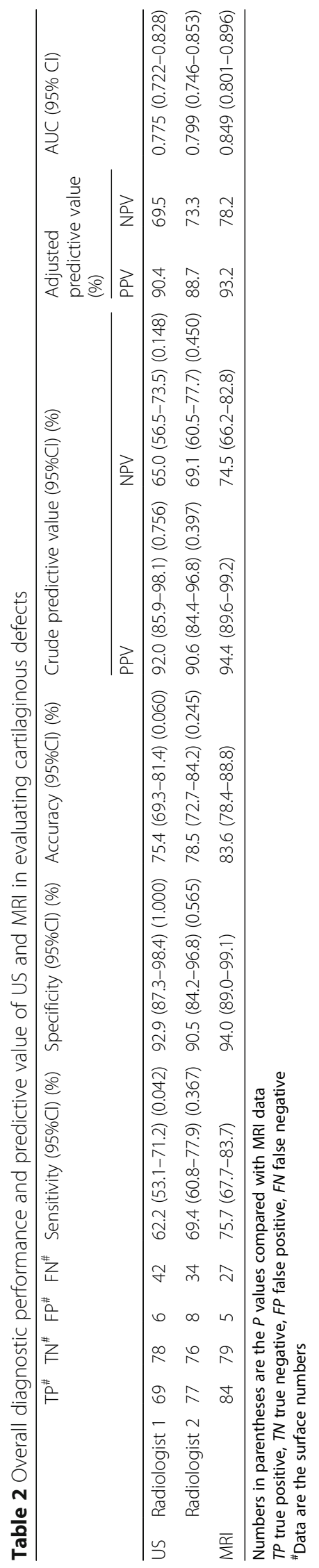




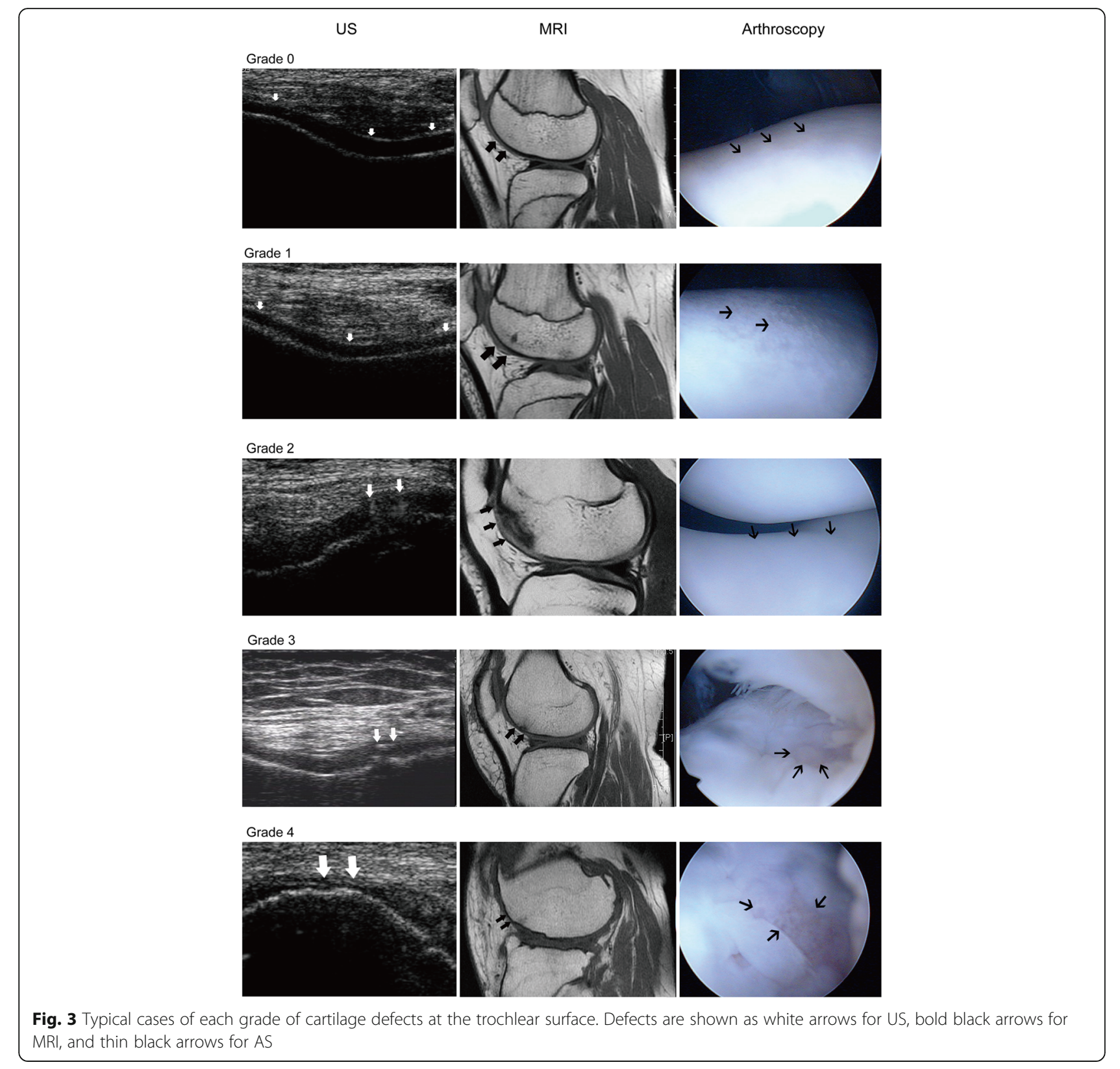

differences between US and MRI ( $P=0.060$ to 1.000$)$, as shown in Table 2.

\section{US and MR diagnosis performance comparisons for different surfaces}

For all cartilage defect grades on each articular surface, some differences were seen between US and MRI for specific surfaces (Table 3), but there were differences between the two radiologists.

\section{US and MR diagnosis performance comparisons for different defect grades}

The respective detection rates and comparisons between US and MRI for cartilage defects of each grade are shown in Tables 4 and 5. Generally, compared with MRI, no significant differences were obtained in detecting grades 0,2 , 3 , and 4 defects $(P=0.083$ to 0.317$)$, but a lower detection rate with US for grade 1 defects was obtained for both radiologists $(P=0.025)$. Indeed, for radiologist 1 , ten lesions were diagnosed as grade 0 , eight as grade 2 , and two as grade 3 . For radiologist 2, eight lesions were diagnosed as grade 0 , six as grade 2 , and six as grade 3 . The proportions of cartilage defects graded identically and matched within one grade of arthroscopic values using US by the two radiologists were 80.0 and $80.5 \%$, respectively, with no significant difference between both radiologists $(P=0.179$ and $P$ $=0.224$, respectively). A moderate agreement between arthroscopy and US for grade assignment (weighted $\kappa=$ 


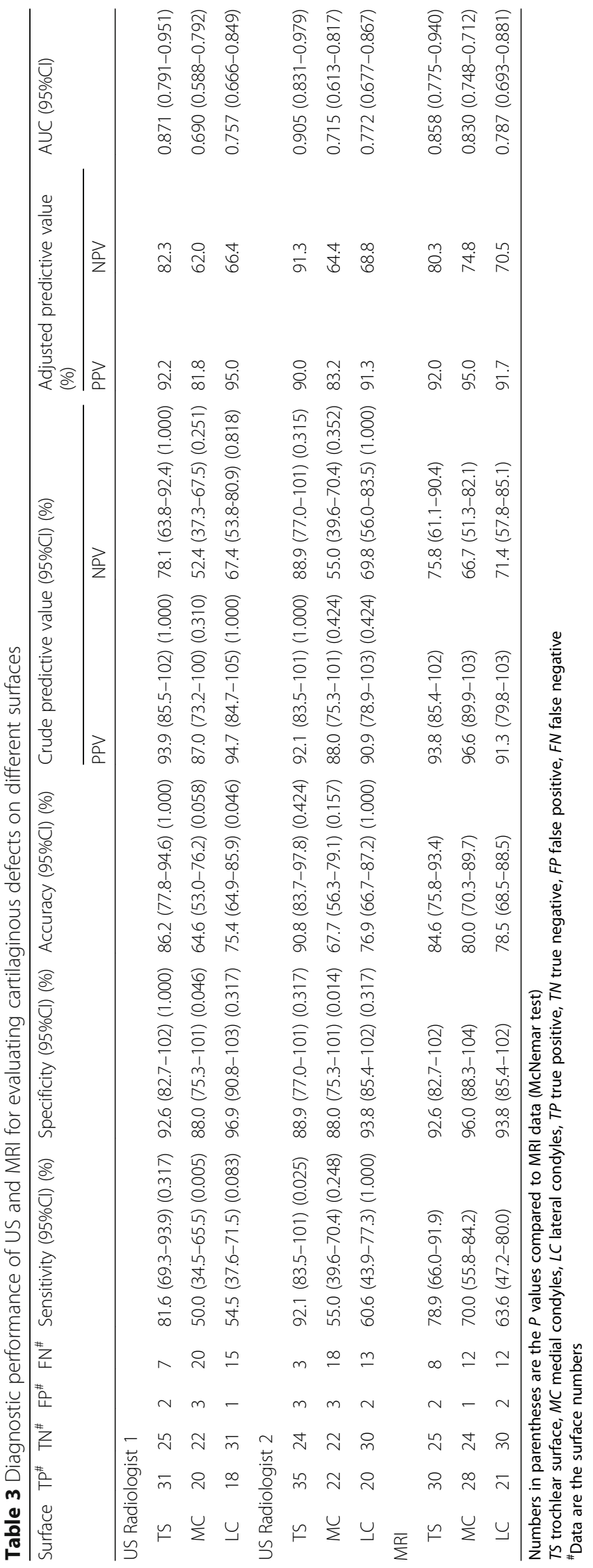


Table 4 Results of US and MR imaging for detecting knee cartilage defects of each grade

\begin{tabular}{llll}
\hline Grade & US & \multirow{2}{*}{ MRI } \\
\cline { 2 - 3 } & Radiologist 1 & Radiologist 2 & \\
\hline 0 & $92.9(78 / 84)(0.317)$ & $90.5(76 / 84)(0.083)$ & $94.0(79 / 84)$ \\
1 & $0.0(0 / 20)(0.025)$ & $0.0(0 / 20)(0.025)$ & $25.0(5 / 20)$ \\
2 & $33.3(10 / 30)(0.083)$ & $40.0(12 / 30)(0.317)$ & $43.3(13 / 30)$ \\
3 & $55.3(21 / 38)(0.014)$ & $65.8(25 / 38)(0.157)$ & $71.1(27 / 38)$ \\
4 & $73.9(17 / 23)(0.083)$ & $82.6(19 / 23)(0.317)$ & $87.0(20 / 23)$ \\
\hline
\end{tabular}

Data are the percentages, followed by the raw data; numbers in parentheses are the $P$ values in comparison with MRI (McNemar test)

$0.60,95 \%$ CI $0.50-0.71$, and $0.63,95 \%$ CI $0.52-0.74$, for the two radiologists) was obtained, with no significant difference ( $P=0.57$ and $P=0.11$, respectively) in comparison with MRI data (weighted $\kappa=0.73$, 95\% CI 0.62-0.84). In addition, substantial agreement was found between grades assigned using US and MRI (weighted $\kappa=0.61,95 \%$ CI $0.50-0.72)$.

\section{Reasons for false-positive and false-negative diagnoses} Error analysis was carried out to determine the reasons for false-positive and false-negative diagnoses by US, and the results are shown in Table 6. The majority of false negatives were due to the lesions being located at certain sites, including the femoral condyles near the intercondyloid fossa, where no defects were detected by US (Fig. 4). The majority of false positives were attributed to the partial volume effect (Fig. 5).

\section{Discussion}

In this study, we aimed to improve the diagnostic accuracy of US for the diagnosis of knee lesions with the intention of improving the accessibility and decreasing the costs of knee examination associated with MRI and arthroscopy, particularly for outpatients. Nevertheless, MRI and arthroscopy are still necessary. Therefore, this study assessed the diagnostic value of a novel US scanning approach in evaluating knee femoral cartilaginous defects and found that it allows a similar diagnostic

Table 5 Comparison of aberrations between US and MRI grades and surgical grades

\begin{tabular}{lllll}
\hline & Modality & US radiologist 1 & US radiologist 2 & MRI \\
\hline Undergrading & $\begin{array}{l}2-4 \\
\text { grades }\end{array}$ & $29(14.9)$ & $14(7.2)$ & $19(9.8)$ \\
& 1 grade & $18(9.2)$ & $12(6.1)$ & $14(7.2)$ \\
Identical & - & $126(64.6)$ & $132(67.7)$ & 144 \\
grading & & & & $(73.8)$ \\
Overgrading & 1 grade & $12(6.2)$ & $13(6.7)$ & $9(4.6)$ \\
& $\begin{array}{l}2-4 \\
\text { grades }\end{array}$ & $10(5.1)$ & $24(12.3)$ & $9(4.6)$ \\
\hline
\end{tabular}

Data are the number of defects. Values in parentheses are percentages
Table 6 Error analysis: reasons for false positive and false negative diagnoses

\begin{tabular}{lllllll}
\hline Surface & \multicolumn{2}{l}{ False-negative finding } & & \multicolumn{2}{l}{ False-positive finding ${ }^{\#}$} \\
\cline { 2 - 4 } & $\begin{array}{l}\text { Particular } \\
\text { sites }\end{array}$ & $\begin{array}{l}\text { Partial } \\
\text { volume } \\
\text { effect }\end{array}$ & $\begin{array}{l}\text { Small or } \\
\text { superficial } \\
\text { lesion }\end{array}$ & $\begin{array}{l}\text { Thin } \\
\text { cartilage }\end{array}$ & $\begin{array}{l}\text { Partial } \\
\text { volume } \\
\text { effect }\end{array}$ \\
\hline TS & $0 / 0$ & $3 / 3$ & $4 / 3$ & $0 / 0$ & $2 / 3$ \\
MC & $9 / 7$ & $5 / 4$ & $6 / 5$ & & $1 / 1$ & $2 / 3$ \\
LC & $11 / 9$ & $4 / 3$ & $0 / 0$ & & $0 / 0$ & $1 / 1$ \\
Total & $20 / 16$ & $12 / 10$ & $10 / 8$ & & $1 / 1$ & $5 / 7$
\end{tabular}

TS trochlear surface, $M C$ medial condyles, $L C$ lateral condyles

"Data are the surface numbers for radiologist 1/radiologist 2

performance as routine MRI, but with improved NPV compared with previous US scanning approach, which is of clinical significance. Different articular surfaces of the femur can be accessible with an external US probe by varying the angle range of knee flexion [23-25]. Interestingly, the novel scanning approach had similar sensitivity, specificity, and accuracy compared with MRI for detecting lesions on the whole femoral cartilage and individual articular surfaces. In addition, moderate agreement was obtained between grades assigned by arthroscopy and US.

In the present study, a substantial interobserver agreement was observed, while similar PPVs and higher NPVs were obtained in comparison with recent reports [13, 14]. The improved NPV (i.e., decreased false-negative rate) could be a consequence of more visibility of the femoral cartilage in the novel scanning approach. The fairly low NPV reported by Saarakkala et al. [14] is probably not related to an intrinsic limitation of the US itself or a need for higher resolution imaging but rather a lack of thorough observation of the overall femoral cartilage. An available acoustic window is the most important factor for US examination. The difficulty in visualizing the whole femoral cartilage due to the shadow of the patella and tibia is a major disadvantage. By using multiple knee angles, the novel approach markedly decreased false-negative femoral cartilage defect diagnoses.

In full extension $\left(0^{\circ}\right)$, the patella rests over the supratrochlear fat pad and is almost completely proximal to the superior border of the femoral articular cartilage [22, 23]. In this position, US can show the cartilage of the anterior and posterior femoral condyles but not the trochlear surface and weight-bearing femoral condyles due to the interference of the patella and tibia. In $10^{\circ}-20^{\circ}$ flexion, the patella first hugs the femoral shaft closely then slips distally and always remains in contact with the trochlear surface of the femur [22, 23], which may lead to poor visibility of most parts of the femoral articular surfaces. At approximately $135^{\circ}$ of flexion, the patella reaches as far as the intercondylar notch [23]. At this time, good exposure 


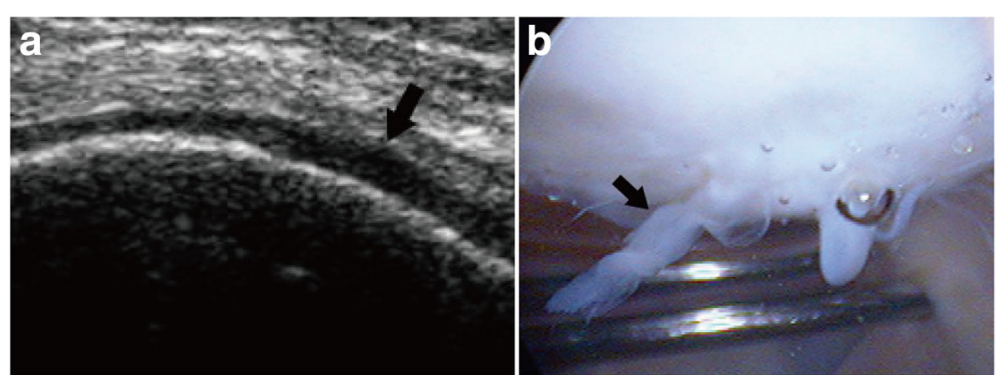

Fig. 4 A grade 2 lesion at the lateral femoral condyles near the intercondyloid fossa, missed by US. a On the US, the diagnosis was normal (grade 0), but on arthroscopy $\mathbf{b}$, the diagnosis was grade 2 (black arrow) cartilage defect, presenting as a velvet-like formation with intact cartilage surface, located at the lateral femoral condyles near the intercondyloid fossa

of the entire trochlear surface and most parts of the lateral and medial condyles can be achieved. Therefore, scanning on minimum $\left(0^{\circ}\right)$ and maximum $\left(\geq 135^{\circ}\right)$ angles of the knee flexion may provide a more thorough scan of the femoral cartilage than those using only a fixed flexed knee (e.g., $\left.120^{\circ}\right)$.

MRI is considered a method of choice for thorough evaluation of cartilage morphology, but its routine use in all symptomatic patients with clinical suspicion of knee cartilage defects is limited due to unavailability in many district and community hospitals in China and high costs (in terms of money and time) [26]. Therefore, the application of the simple, widely available, and inexpensive US technique as the initial screening method for femoral cartilage lesions could be more appropriate. The novel US approach proposed here may satisfy the above requirements and can be used as an initial screening modality to provide a morphological assessment of the femoral articular cartilage in outpatient clinics.

As a non-invasive imaging modality, the novel approach needed further clinical validation. Therefore, the novel approach was compared with MRI, which is probably the most important method for cartilage imaging [26]. Previous comparative studies [27, 28] between US and MRI mainly focused on the thickness measurement of femoral cartilage, an important defect indicator, and showed a significant correlation (coefficients $=0.44-$ 0.84). Comparison of US and MRI was further assessed in the present study; although a relatively lower detection rate of grade 1 defects was observed, the novel approach showed similar diagnostic ability for the detection and classification of cartilage defects compared with routine 2D FSE MRI, with a significant agreement for grading lesions.

The first major problem is that although the novel approach allows a significant decrease of false-negative diagnoses, it should be highlighted that the risk of false negatives was still as high as $26.7-30.5 \%$, representing the majority of erroneous diagnoses. This likely results from the inability to visualize the lateral and medial condyles near the intercondylar notch, even at the maximum angle (e.g., $135^{\circ}$ ) of the knee flexion, due to their continuous articulation with the lateral and odd facet of the patella [15]. Thus, defects in these locations were the major cause of false negatives, as none of them was detected. Therefore, the blind areas of US have been improved by using varying flexion $\left(0-135^{\circ}\right)$ rather than a fixed flexion $\left(120^{\circ}\right)$, but the novel approach still needs to be improved. Patients suspected to be with cartilage defects should undergo additional diagnostic modalities, e.g., MRI, even with a negative US finding to verify the cartilage status.
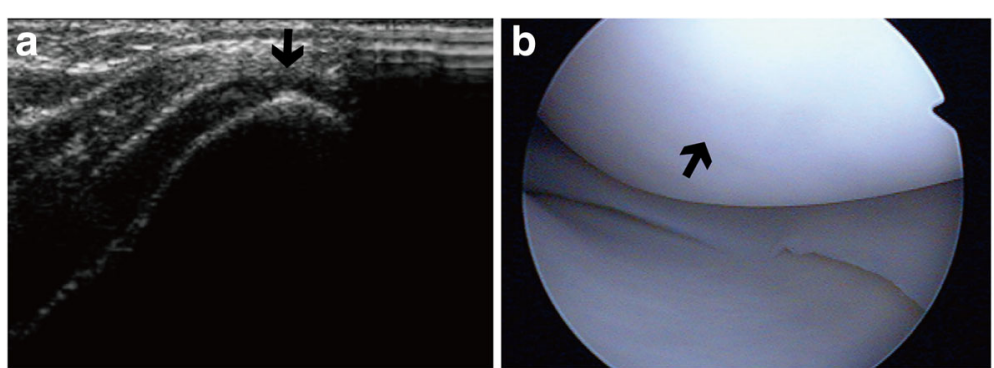

Fig. 5 Example of a false-positive case. a The case was diagnosed as grade 3 cartilage defect on the trochlear surface (black arrow), presenting as blurred margin, lack of clarity, and overt local thinning (> 50\% of cartilage depth). b On AS, the diagnosis was grade 0, i.e., normal cartilage (black arrow) 
The second major problem is that only femoral surfaces can be seen by US, not the patellar and tibial surfaces, which precludes the technique from providing an overall assessment of the knee articular cartilage. Nevertheless, strong correlations (Pearson's correlation coefficients $=0.75-0.77$ ) between volume changes in femoral cartilage and that in tibial cartilage in OA patients have been reported [29], indicating that evaluating one of the two features should be adequate. Therefore, US findings from the femoral cartilage might be reliable for evaluating arthritic cartilage changes of the tibial cartilage in clinical practice.

The US systems and technique are possible sources of error in US, as well as the operator. Although similar diagnostic accuracy between the novel approach and MRI was presented here, a substantial number of patients with small or superficial lesions (grade 1 defects) were misdiagnosed or missed by the US. Indeed, the US equipment available for routine clinical use can only assess conspicuous morphological changes of cartilage, not determining its internal characteristics, while MRI can. Therefore, subtle morphological changes in the early stage of cartilage defects might explain the misdiagnoses. More advanced equipment and techniques (e.g., a 50- $\mathrm{MHz}$ transducer, which can detect layers in immature cartilage [30]) may provide a solution. Further studies are necessary to verify this hypothesis. Another issue is operator dependency, a known problem in US examination [31]. As shown above, the difference in overall sensitivity was obtained between the two radiologists participating in this study. Therefore, a standardized training to learn the correct scanning approach and associated diagnoses is essential to avoid misdiagnoses or missed diagnoses.

There were some limitations to this study. First, although routine 2D FSE MRI sequences were performed as previously described [32-34], it may be argued that this study underestimated the actual diagnostic ability of MRI, as it is not optimal for cartilage evaluation due to anisotropic voxels, section gaps, and partial volume effects [26]. In addition, several MRI techniques are available to facilitate the assessment of the femoral cartilage for changes of morphology [35-37] and even biochemical composition [38, 39]. The results of MRI achieved in such sequences may be more favorable than those reported here. Nevertheless, since the cause of pain or disability of the knee is frequently multifactorial or unknown, 2D FSE sequences are most commonly applied in the clinical setting for initial examinations. In this study, we simulated a hypothetical situation of the first-time examination, which optimized the likelihood of screening cartilage defects.

Another limitation is that the same cartilage lesion could be attributed to different sites between US and MRI or arthroscopy. To minimize such discrepancies, the same standard schematic drawing of the femoral cartilage surfaces was used for all techniques. Nevertheless, a lesion located on the very edge of three articular surfaces would be likely assigned to different surfaces in various examination methods. Therefore, an overall assessment of the femoral cartilage from all three sites is necessary before the diagnosis and subsequent treatment of cartilage lesion; this is not affected by the possible misplacement. Further studies regarding treatment evaluation are required to target the precise lesion localization of cartilage.

In addition, the correlation between US findings and other assessment tools was not established. Indeed, in this initial study, we prioritized the associations of US with arthroscopy (gold standard) and MRI (most important imaging modality of cartilage). In the future, the correlation between the novel US scanning approach and clinical assessment should be evaluated for its recommendation in routine clinical use, including evaluation of degenerative changes and therapeutic effects.

The aim of the present study was to investigate the value of US as a screening tool for cartilage defects in patients with a chief complaint of knee pain (without any previous examination and diagnosis). It is indeed possible that some patients were not definitely diagnosed with OA. On the other hand, cartilage degeneration caused by OA may also present as cartilage defect. Therefore, it could be hypothesized that OA will not directly affect the capability of the US detection of cartilage defects, but this specific point will have to be examined in the future.

\section{Conclusions}

The novel US scanning approach taking knee articular motion into consideration is more valid in a clinical setting to significantly decrease false-negative diagnoses compared with fixed-angle $\left(120^{\circ}\right)$ transverse scanning. It also has similar diagnostic performance, PPV, and agreement as routine MRI approaches for evaluating the knee cartilage defects in patients with a broad spectrum of knee diseases, but the NPV was higher than the previous US scanning approach. As a non-invasive, fast, inexpensive, and radiation-free imaging modality, US has a potential to be used for initial screening assessments of cartilage defects in first-visit patients with a chief complaint of knee pain and/or disability.

\section{Additional file}

Additional file 1: Table S1. Parameters for MRI sequences. (DOCX 15 kb)

\section{Abbreviations}

fs: Fat-saturated; LC: Lateral femoral condyles; MC: Medial femoral condyles; MRI: Magnetic resonance imaging; NPV: Negative predictive values; OA: Osteoarthritis; PD: Proton density; PPV: Positive predictive value; TS: Trochlear surface; US: Ultrasound 


\section{Acknowledgements}

The authors acknowledge the invaluable participation of the patients as well as the help from the Radiology and Orthopedics Departments of the Third Affiliated Hospital of Sun Yat-Sen University and Dr. Jibin Liu of Department of Radiology, Thomas Jefferson University Hospital.

\section{Availability of data and materials}

The datasets generated during the current study are not publically available due to ethical restrictions but are available from the corresponding author on reasonable request.

\section{Authors' contributions}

$J Y C, B W Z$, and JR contributed to the conception and design; JYC, BWZ, XCM, $\mathrm{YL}, \mathrm{HDL}, \mathrm{KW}, \mathrm{DMH}$, and JR contributed to the acquisition of data or analysis and interpretation of data; JYC, BWZ, XCM, YL, HDL, KW, DMH, and JR have been involved in the drafting of the manuscript and revised it critically for important intellectual content; all authors have given final approval of the version to be published.

\section{Ethics approval and consent to participate}

The study was approved by the research ethics committee of the Third Affiliated Hospital of Sun Yat-sen University. Informed consent was obtained from all individual participants included in the study.

\section{Consent for publication}

Informed consents for image publication were taken from all the patients and one of the authors, Bowen Zheng.

\section{Competing interests}

The authors declare that they have no competing interests.

\section{Publisher's Note}

Springer Nature remains neutral with regard to jurisdictional claims in published maps and institutional affiliations.

\begin{abstract}
Author details
${ }^{1}$ Department of Medical Ultrasonics, The Third Affiliated Hospital of Sun Yat-sen University, 600 Tianhe Road, Guangzhou 510630, People's Republic of China. ${ }^{2}$ Department of Radiology, The Third Affiliated Hospital of Sun Yat-sen University, 600 Tianhe Road, Guangzhou 510630, People's Republic of China. ${ }^{3}$ Department of Orthopedics, The Third Affiliated Hospital of Sun Yat-sen University, 600 Tianhe Road, Guangzhou 510630, People's Republic of China.
\end{abstract}

Received: 17 December 2017 Accepted: 9 July 2018

Published online: 16 July 2018

\section{References}

1. Urwin M, Symmons D, Allison T, Brammah T, Busby H, Roxby M, et al. Estimating the burden of musculoskeletal disorders in the community: the comparative prevalence of symptoms at different anatomical sites, and the relation to social deprivation. Ann Rheum Dis. 1998:57:649-55.

2. Buckwalter JA, Mankin HJ. Articular cartilage repair and transplantation. Arthritis Rheum. 1998:41:1331-42.

3. Qvistgaard E, Kristoffersen H, Terslev L, Danneskiold-Samsoe B, TorpPedersen S, Bliddal H. Guidance by ultrasound of intra-articular injections in the knee and hip joints. Osteoarthr Cartil. 2001;9:512-7.

4. Wakefield RJ, Gibbon WW, Emery P. The current status of ultrasonography in rheumatology. Rheumatology (Oxford). 1999;38:195-8.

5. McCune WJ, Dedrick DK, Aisen AM, MacGuire A. Sonographic evaluation of osteoarthritic femoral condylar cartilage. Correlation with operative findings. Clin Orthop Relat Res. 1990;254:230-5.

6. lagnocco A, Coari G, Zoppini A. Sonographic evaluation of femoral condylar cartilage in osteoarthritis and rheumatoid arthritis. Scand J Rheumatol. 1992; 21:201-3.

7. Grassi W, Lamanna G, Farina A, Cervini C. Sonographic imaging of normal and osteoarthritic cartilage. Semin Arthritis Rheum. 1999;28:398-403.

8. Batalov AZ, Kuzmanova SI, Penev DP. Ultrasonographic evaluation of knee joint cartilage in rheumatoid arthritis patients. Folia Med (Plovdiv). 2000;42:23-6.
9. Disler DG, Raymond E, May DA, Wayne JS, McCauley TR. Articular cartilage defects: in vitro evaluation of accuracy and interobserver reliability for detection and grading with US. Radiology. 2000;215:846-51.

10. Mathiesen $\mathrm{O}$, Konradsen L, Torp-Pedersen S, Jorgensen U. Ultrasonography and articular cartilage defects in the knee: an in vitro evaluation of the accuracy of cartilage thickness and defect size assessment. Knee Surg Sports Traumatol Arthrosc. 2004;12:440-3.

11. Tsai CY, Lee CL, Chai CY, Chen CH, Su JY, Huang HT, et al. The validity of in vitro ultrasonographic grading of osteoarthritic femoral condylar cartilage-a comparison with histologic grading. Osteoarthr Cartil. 2007;15:245-50.

12. Yoon $\mathrm{CH}$, Kim HS, Ju JH, Jee WH, Park SH, Kim HY. Validity of the sonographic longitudinal sagittal image for assessment of the cartilage thickness in the knee osteoarthritis. Clin Rheumatol. 2008;27:1507-16.

13. Lee $\mathrm{CL}$, Huang MH, Chai CY, Chen CH, Su JY, Tien YC. The validity of in vivo ultrasonographic grading of osteoarthritic femoral condylar cartilage: a comparison with in vitro ultrasonographic and histologic gradings. Osteoarthr Cartil. 2008;16:352-8.

14. Saarakkala S, Waris P, Waris V, Tarkiainen I, Karvanen E, Aarnio J, et al. Diagnostic performance of knee ultrasonography for detecting degenerative changes of articular cartilage. Osteoarthr Cartil. 2012;20: 376-81.

15. Shahriaree H. O'Connor's textbook of arthroscopic surgery. Philadelphia: Lippincott; 1992.

16. Li Y, Hua Y, Fang J, Wang C, Qiao L, Wan C, et al. Performance of different scan protocols of fetal echocardiography in the diagnosis of fetal congenital heart disease: a systematic review and meta-analysis. PLoS One. 2013;8:e65484

17. Dexheimer Neto FL, Andrade JM, Raupp AC, Townsend Rda S, Beltrami FG, Brisson $\mathrm{H}$, et al. Diagnostic accuracy of the bedside lung ultrasound in emergency protocol for the diagnosis of acute respiratory failure in spontaneously breathing patients. J Bras Pneumol. 2015;41:58-64.

18. Nazerian P, Tozzetti C, Vanni S, Bartolucci M, Gualtieri S, Trausi F, et al. Accuracy of abdominal ultrasound for the diagnosis of pneumoperitoneum in patients with acute abdominal pain: a pilot study. Crit Ultrasound J. 2015;7:15

19. Brittberg M, Winalski CS. Evaluation of cartilage injuries and repair. J Bone Joint Surg Am. 2003;85-A(Suppl 2):58-69.

20. Aisen AM, McCune WJ, MacGuire A, Carson PL, Silver TM, Jafri SZ, et al. Sonographic evaluation of the cartilage of the knee. Radiology. 1984; 153:781-4.

21. Altman DG, Bland JM. Diagnostic tests 2: predictive values. BMJ. 1994; 309:102.

22. Kundel HL, Polansky M. Measurement of observer agreement. Radiology. 2003;228:303-8

23. Jiang F, Xu B, Zhang XS, Wang L, Wen CJ. Feasibility of the femoral condylar cartilage ultrasound imaging. Acta Univ Med Anhui. 2003;38:144-6.

24. Outerbridge RE. The etiology of chondromalacia patellae. J Bone Joint Surg Br. 1961;43-B:752-7.

25. Goodfellow J, Hungerford DS, Zindel M. Patello-femoral joint mechanics and pathology. 1. Functional anatomy of the patello-femoral joint. J Bone Joint Surg Br. 1976:58:287-90.

26. Roemer FW, Crema MD, Trattnig S, Guermazi A. Advances in imaging of osteoarthritis and cartilage. Radiology. 2011:260:332-54.

27. Ostergaard M, Court-Payen M, Gideon P, Wieslander S, Cortsen M, Lorenzen I, et al. Ultrasonography in arthritis of the knee. A comparison with MR imaging. Acta Radiol. 1995;36:19-26.

28. Tarhan S, Unlu Z. Magnetic resonance imaging and ultrasonographic evaluation of the patients with knee osteoarthritis: a comparative study. Clin Rheumatol. 2003;22:181-8.

29. Cicuttini FM, Wluka AE, Stuckey SL. Tibial and femoral cartilage changes in knee osteoarthritis. Ann Rheum Dis. 2001;60:977-80.

30. Kim HK, Babyn PS, Harasiewicz KA, Gahunia HK, Pritzker KP, Foster FS. Imaging of immature articular cartilage using ultrasound backscatter microscopy at 50 MHz. J Orthop Res. 1995:13:963-70.

31. Roemer FW, van Holsbeeck M, Genant HK. Musculoskeletal ultrasound in rheumatology: a radiologic perspective. Arthritis Rheum. 2005:53:491-3.

32. Kijowski R, Blankenbaker DG, Davis KW, Shinki K, Kaplan LD, De Smet AA Comparison of 1.5- and 3.0-T MR imaging for evaluating the articular cartilage of the knee joint. Radiology. 2009;250:839-48.

33. Kijowski R, Blankenbaker DG, Woods MA, Shinki K, De Smet AA, Reeder SB. 3.0-T evaluation of knee cartilage by using three-dimensional IDEAL 
GRASS imaging: comparison with fast spin-echo imaging. Radiology. 2010;255:117-27.

34. Duc SR, Pfirrmann CW, Schmid MR, Zanetti M, Koch PP, Kalberer F, et al. Articular cartilage defects detected with 3D water-excitation true FISP: prospective comparison with sequences commonly used for knee imaging. Radiology. 2007;245:216-23.

35. Disler DG, McCauley TR, Wirth CR, Fuchs MD. Detection of knee hyaline cartilage defects using fat-suppressed three-dimensional spoiled gradientecho MR imaging: comparison with standard MR imaging and correlation with arthroscopy. AJR Am J Roentgenol. 1995;165:377-82.

36. Eckstein F, Hudelmaier M, Wirth W, Kiefer B, Jackson R, Yu J, et al. Double echo steady state magnetic resonance imaging of knee articular cartilage at 3 Tesla: a pilot study for the osteoarthritis initiative. Ann Rheum Dis. 2006; 65:433-41.

37. Duc SR, Koch P, Schmid MR, Horger W, Hodler J, Pfirrmann CW. Diagnosis of articular cartilage abnormalities of the knee: prospective clinical evaluation of a 3D water-excitation true FISP sequence. Radiology. 2007;243:475-82.

38. Kim YJ, Jaramillo D, Millis MB, Gray ML, Burstein D. Assessment of early osteoarthritis in hip dysplasia with delayed gadolinium-enhanced magnetic resonance imaging of cartilage. J Bone Joint Surg Am. 2003;85-A:1987-92.

39. Borthakur A, Shapiro EM, Beers J, Kudchodkar S, Kneeland JB, Reddy R. Sensitivity of MRI to proteoglycan depletion in cartilage: comparison of sodium and proton MRI. Osteoarthr Cartil. 2000;8:288-93.

\section{Ready to submit your research? Choose BMC and benefit from:}

- fast, convenient online submission

- thorough peer review by experienced researchers in your field

- rapid publication on acceptance

- support for research data, including large and complex data types

- gold Open Access which fosters wider collaboration and increased citations

- maximum visibility for your research: over $100 \mathrm{M}$ website views per year

At $\mathrm{BMC}$, research is always in progress.

Learn more biomedcentral.com/submissions 